\title{
Perceived Barriers to Maintaining Healthy Body Weight among Saudi Women of Reproductive Age in Jeddah City
}

\author{
Alharbi $\mathrm{MM}^{*}$ and Robert T Jackson \\ Department of Nutrition and Food Science, Skinner Hall, University of Maryland, College Park, MD, USA \\ *Corresponding author: Alharbi MM, Department of Nutrition and Food Science, 0119C Skinner Hall, \\ University of Maryland, College Park, MD USA 20742-7521, Tel: 240-463-5044, E-mail: malharbi.sa@gmail. \\ com
}

Citation: Alharbi MM, Robert T Jackson (2017) Perceived Barriers to Maintaining Healthy Body Weight among Saudi Women of Reproductive Age in Jeddah City. J Obes Overweig 3(2): 202. doi: 10.15744/2455-7633.3.202

Received Date: July 20, 2017 Accepted Date: November 27, 2017 Published Date: November 29, 2017

\begin{abstract}
Background: Maintaining a healthy body weight is important for overall health and can help prevent and control many chronic conditions. However, the information surrounding the barriers to healthy eating (HE) and physical activity (PA) for weight maintenance among Saudi women of reproductive age is insufficient to design and develop intervention programs.

Objective: To explore personal, social, and physical environmental factors that act as barriers to maintaining a healthy weight and how these barriers vary by socio-demographic and weight status.

Methods: A cross-sectional study was conducted in 2014 using a stratified two-stage cluster sampling design comprising 408 Saudi women attending 12 Jeddah Primary Health Centers (JPHCCs). Data was collected using a structured questionnaire consisting of sociodemographic factors, eating habits (EHs), PA, and perceived barriers to a maintenance of healthy weight maintenance. Body mass index (BMI) and waist circumference (WC) data also were obtained.

Results: Of the 408 women evaluated, $33.8 \%$ were obese $\left(B M I \geq 30 \mathrm{~kg} / \mathrm{m}^{2}\right), 25.1 \%$ were abdominally obese with WC $\geq 88 \mathrm{~cm}, 24 \%$ had unhealthy EHs, and $31.2 \%$ were physically inactive. A high proportion of women faced significant barriers in maintaining their weight related to EHs or PA (49.2\% vs. 50.7\%). The most common barriers to HE and PA in the study group were lack of willpower, skills, knowledge, enjoyment, time, resources, and social influence. Social norms and hot weather had a great impact on the women's PA levels. There was a positive significant association between EHs and PA and between EHs and PA barriers, but no significant associations were found between barriers to maintaining healthy weight and either type of obesity. The personal and the social environmental barriers to HE had a significant negative association with EHs, while none of the examined barriers to PA were associated with PA. There were inversely significant associated between the HE barrier and age, and between PA barrier and income status.
\end{abstract}

Conclusion: The study findings suggest there is a need to improve women's EHs and PA by eliminating the identified barriers. These findings can be used to develop appropriate and effective obesity prevention interventions for Jeddah women.

Keywords: Obesity; Health barriers; Physical activity; Eating habits; Saudi; Reproductive-aged women

List of Abbreviations: BMI: Body Mass Index; WC: Waist Circumference; PHCCs: Primary Health Care Centers; PA: Physical Activity; EHs: Eating Habits; HE: Healthy Eating; PSU's: Primary Sampling Units; PPS: Probabilities Proportional to their Size; IPAQ: International Physical Activity Questionnaire; MET-Min/week: Metabolic Equivalent Task Minutes per week; WHO: World Health Organization standards

\section{Introduction}

Obesity and unhealthy lifestyle choices are growing problems associated with major health issues. Obesity is one of the ten leading health indicators used to measure health status [1]. The prevalence of obesity in Saudi Arabia has been shown to be significantly higher among women (33.5\%) than men (24.1\%) [2]. Also, Saudi women had a high rate of chronic disease: hypertension (12.5\%), diabetes (11.7\%), and hypercholesterolemia (7.3\%) [3]. Despite the high rate of obesity among Saudi women, there have been limited studies conducted to understand the barriers women perceive in attempting to maintain a healthy weight.

Maintaining a healthy body weight is important for overall health and can help prevent and control obesity and its associated diseases [4]. Healthy eating (HE) and physical activity (PA) are vital strategies for losing and maintaining weight. The perceived barriers to increasing PA and improving HE that women face may vary according to their social and personal circumstances [5]. Barriers are defined as factors that impede health-promoting behaviors and include perceptions about the potentially negative 
effects of changing. Health-promotion and disease-prevention literature have established barriers as important predictors of behavior change [6]. Generally, weight management results from many impediments to PA and HE, including those related barriers to personal (e.g., lack of willpower, knowledge, motivation, cooking skills, and exercise), social environmental (e.g., social influence, family support, and commitment), and physical environmental (e.g., lack of money, limited access to exercise facilities, and a hot climate) [7]. Moreover, young women are more likely than older women to experience particular life events, such as leaving the family home, starting work, entering marriage, and becoming mothers, that may influence their PA and eating habits (EHs) [5].

Diet and nutrition play important roles in maintaining health and preventing obesity and numerous diseases [4]. A decrease in morbidity and mortality associated with lifestyle health related diseases may be achievable if healthy EHs are adopted early in life and maintained in the long term [8]. HE reflects a complex decision-making process influenced by numerous factors or barriers, including demographic, social, personal, and emotional [9]. With a view on informing policy, a recent study was conducted by Al-Jaaly (2011) to quantify the problem of overweight and obesity in adolescent girls (13-18 years old) in Jeddah City [10]. The AlJaaly (2011) study indicated a strong association between a number of factors and weight status of adolescent Jeddah girls. These factors included individual elements, such as biological factors (e.g., age of menarche), EHs, lifestyle, and environmental factors like family influence, access to food, and societal influence. There is limited research, however, on EHs and related barriers in Saudi adult women for maintaining a healthy body.

PA is also an important component of a healthy lifestyle and has been described as an essential factor in managing many health conditions and combating the obesity epidemic [11,12]. Several studies have shown that engaging in PA on a regular basis (at least 30 minutes of moderate PA per day) enhances the quality of life and health. Additionally, PA helps prevent and control obesity, type 2 diabetes, cardiovascular diseases, and hypertension [13]. In Saudi Arabia, sedentary lifestyles and low levels of PA have become the norm among the Saudi population [14]. According to data from the 2013 Saudi National Health Survey, the prevalence of physical inactivity was high among the Saudi population (15 years of age and older), revealing significantly higher levels of inactivity in women (75.1\%) than in men (47.0\%) [2]. Such levels of inactivity could be caused by the numerous challenges Saudi women face to being physically active, such as being prohibited from driving, hot weather, requiring a guardian for commuting, wearing an abaya (an outer garment worn by Muslim women), or needing the family's permission to engage in PA outside the home (such as walking in a public area or attending a fitness gym) [15]. However, information on the levels of PA and its barriers in Saudi adult women is lacking, especially among women particularly at risk of weight gain, such as those of reproductive age.

It is important to monitor and address adverse weight transitions among women in the stage of their reproductive years, as these transitions will have adverse effects, not only on women's short- and long-term health, but also on that of their children [16]. However, as most studies in Saudi Arabia have focused on the male population, children, adolescents, and women in their college years (under the age of 24 years), existing literature offers insufficient data regarding the perceived barriers to maintaining healthy body weight related to EHs and PA among Saudi women of reproductive age. Moreover, to the best of the authors' knowledge, no study has been conducted to assess EHs, PA, and perceived barriers to following a healthy lifestyle among Saudi women of reproductive age in Jeddah City, the most liberal, urban, and diverse city in Saudi Arabia, where the prevalence of obesity is high and the number of reproductive-age women is increasing [17]. This study was conducted in Jeddah to fill the gap in the literature using a representative sample of Saudi women (15-49 years) who attended services at Jeddah Primary Health Care Centers (JPHCCs) to explore personal, social, and physical environmental factors that act as barriers to maintaining a healthy weight. This study also investigated how these varied by socio-demographic factors, as well as evaluated the relationship between those barriers and EHs, PA, and obesity. Determining these factors and barriers is vital to creating the best programs for combating the prevalence of obesity for not only the women of Jeddah City but also potentially the women of other cities in Saudi Arabia.

\section{Methods}

\section{Sample}

The study was conducted using a cross-sectional stratified two-stage cluster sampling design survey of 408 Saudi women, aged 1549 years, who attended general clinics at JPHCCs. The sampling procedure aimed to select a representative sample of women who were only seeking services at PHCCs in Jeddah City. Clinics served as Primary Sampling Units (PSU's) and were grouped into four health sector strata. In the first stage, the PHCCs (clusters) were sampled without replacement and selected (12 out of 37 centers) with Probabilities Proportional to their Size (PPS), from the list of PHCCs in survey area. The second sampling stage involved recruitment of women from the selected PHCCs (12 centers) using a systematic sampling procedure from the eligible women attending on days the sampling PHCCs (General Clinics) were visited. The first woman participant who fulfilled the inclusion criteria was invited to enroll in the study. Then, every third attending woman who fulfilled the criteria was selected and soon until completion of the required sample from the PHCC was achieved. However, if a selected woman did not fulfill the inclusion criteria (exclusion criteria) or refused to participate in this study, then selection proceeded to the next sample woman attending the PHCC. Subjects who were pregnant/ lactating and those having serious diseases (e.g., organ failure, transplant, ascites, cancer, and mental illness), were excluded.

Sample size was determined by performing an a-priori power analysis to determine the number of participants required to detect a small effect of design $\left(\mathrm{f}^{2}=0.1\right)$ with power $=.80$, and at $\alpha=.05$ (the power analysis was conducted with $\mathrm{G}^{\star}$ Power 3.1.4.). The analysis 
indicated a sample size of 201 would be sufficient. Then, this was adjusted for clustering by multiplying this sample size by a convenient design effect of 2.0, which indicated that a minimum of 402 women would be sufficient to accurately estimate results for the final sample. However, to selected equal number of the women from the selected health centers (12 centers) we increased the sample size to 408 ( 34 women / 12 centers).

\section{Survey}

Participants visited the general clinic were asked to participate in answering the survey questions as volunteers (see the appendix A). The informed consent information was distributed to participants before participating in the study. A structured questionnaire (face-to-face interview) was used in the survey to elicit information on the socio-demographic characteristics, $\mathrm{EHs}, \mathrm{PA}$, and lifestyle information, and perceived barriers to weight maintenance questionnaire.

\section{Anthropometric measurements}

Weight and height were measured on a digital scale with stadiometer (Seca 703 medical scale) (Hamburg, Germany). Weight was recorded to the nearest $100 \mathrm{gm}$ and height to the nearest $0.1 \mathrm{~cm}$. Measurements of participants were collected in a private area (exam room) and instructed them to remove excess clothing, overcoats, Hejab, Abaya, and shoes. BMI was calculated as weight divided by height squared $\left(\mathrm{kg} / \mathrm{m}^{2}\right)$, and was stratified for the purpose of analysis into two categories: non-obese and obese based on World Health Organization standards (WHO) 2012 [18]. For adult ( $\geq 20$ years old), non-obese group was defined as BMI $<25 \mathrm{kgm}^{2}$, and obese group (overweight/obese) as BMI $\geq 25 \mathrm{kgm}^{2}$. For adolescents ( $\leq 19$ years old), non obese group was defined as $\mathrm{BMI}<85^{\text {th }}$ age-specific percentile, and obese group (overweight/obese) as $\mathrm{BMI} \geq 85^{\text {th }}$ age-specific percentile. Online software was used to calculate BMI age-specific percentile from height, weight, age, and sex data, based on WHO reference populations [19,20]. WC was measured at the mid-point between the iliac crest and lowest rib to the nearest $0.1 \mathrm{~cm}$. Then, abdominal obesity was defined as WC $>88 \mathrm{~cm}$ based on Who criteria [21].

\section{Eating habits (EHs) Assessment}

EHs were assessed in the questionnaire by taking a selection of items from the reliable EHs questionnaire while others were generated from the literature with expert advice from nutritionists [22-29]. The EHs section of the survey consisted of 24 questions (items), which were designed to investigate the actual eating behaviors of the participants in the study (see the appendix A, section 4). The items referred to both healthy and unhealthy EHs as well as to behaviors. EHs were operationalized with four variables (EHs section) including: the number of regular meals eaten each day, eating fast food, consuming saturated fat, and overall EHs score. Overall EHs were assessed with a mean composite score of 14 items, which had the following response categories: always, often, sometimes, never. The 7 items of the response categories ranged from always (highest score $=4$ ) to never (lowest score $=1$ ) while the scores of other 7 items were reversed (always $=1$ and never $=4$ ). Non-scored items in this questionnaire ( 9 items) were used to obtain further information on Saudi women's dietary practices and behaviors. The total score (56) was divided into tertiles, where the lowest tertile (score $\leq 33$ ) referred to "inadequate EHs," the medium tertile (score $\geq 34$ to 37) referred to "partially satisfactory EHs" and the highest tertile (score 238 ) referred to "satisfactory EHs" [23]. The eating habits questionnaire (13items) that were used to determine the overall eating habits score) was piloted with 20 women (from PHCCs in Jeddah City) to test the reliability using Cronbach's alpha coefficient. The value of Cronbach's alpha coefficient for eating habits items was 0.648 , indicating an acceptable level of internal consistency [30].

\section{Physical Activity (PA) Assessment and lifestyle}

PA was assessed using the official Arabic short version of the International Physical Activity Questionnaire (IPAQ) [31]. IPAQ was subjected to a reliability and validity study carried out in 14 centers in 12 countries during the year 2000, and demonstrated reasonable test-retest reliability (intra-class correlations range 0.7-0.8 and inter-method validity (median $r_{s}=.67$ ) [32]. The short form of IPAQ (see the appendix A, section 5) has been validated and used by numerous studies among the Saudi Arabia adult population [2,14,15,33-35]. The total weekly PA (Metabolic Equivalent Task minutes per week (MET-Min/week)) was calculated by multiplying the number of minutes spent in each activity category (low, moderate, and vigorous levels) with the specific MET score for each activity. The MET intensity values that were used to score IPAQ questions were: vigorous (8 METs), moderate (4 METs) and low (3.3 METs) [36]. For descriptive analysis, the PA scoring was categorized as: physically inactivate (low activity $<600$ MET-min./week), and physically active (sufficient activity $\geq 600$ MET-min./week). Moreover, we asked the participants additional questions to obtain further information on their lifestyles, and how these activities and behaviors affect their body weights.

\section{Perceived barriers to weight maintenance questionnaire}

The barriers questionnaire was designed to provide extensive data about the perceived barriers that Saudi women face in healthy eating (HE) and being physically active. Study participants were presented with a list of 91 possible barriers (items), 41 items to identify the HE barriers set, and 50 items to identify PA barriers set [See Appendix B]. The participants were inquired to select those that would be perceived as presenting major difficulties when trying to maintain their body weight. Participants were asked, 'How important are the following as barriers to maintaining a healthy body weight?' Most of the items included in the questionnaire were adopted from previous research [5,7,37-44]. 
The perceived barrier items formed different groupings around the major barrier themes in the literature, including those related to HE (41 items) and those related to PA (50 items). Each set of perceived barriers had three main categories, namely, personal, social environmental, and physical environmental barriers. The personal barriers for PA were grouped into 8 subsets: lack of willpower, of self-confidence, of skills, of knowledge, of energy, of enjoyment, fear of injury, and health problems, while the personal barriers for HE were grouped into 4 subsets: lack of willpower, of knowledge, of skill, of enjoyment (e.g., does not enjoy eating healthy foods such as low salt, low sugar and fat diet, and following a meal plan would take the pleasure out of eating). The social environmental barriers for PA were grouped into 4 subsets: lack of support, lack of time, social influence, and social norms. Social influence is defined as "change in an individual's thoughts, feelings, attitudes, or behaviors that result from interaction with another individual or a group," while social norms are the rules for how people should act in a given group or society. Any behavior that is outside these norms is considered abnormal [45]. The social environmental barriers to HE were grouped into 4 categories: lack of social support, lack of time, social influence, and lifestyle changes. The physical environmental barriers for PA were grouped into 3 subsets: lack of resources (e.g., lack of money, limited access to exercise facilities, and safe neighborhood areas), lack of transportation, and hot weather. The physical environmental barriers to HE had one subset: lack of resources (e.g., lack of money, food availability, and cooking facilities). Each category consists of two or more items and rates of questions were summed up to find the score of the category.

All barriers on the questionnaire were scored on a 4-point Likert scale that ranged from "very likely" (3) to "very unlikely" (0) [46]. All barriers items were positive statements, which meant that the higher the score, the higher the likelihood that the item was a barrier. Then, the sum-scores of the categories' barriers and subgroups were computed to define the overall barriers sets scores, from adding the sum-scores of personal, social environment, and physical environment barriers together. The median split method was then used to divide respondents into high-scoring and low-scoring groups (important and not important barriers) [47].

The barriers questionnaire was piloted with 20 women (from PHCCs in Jeddah City) to test the reliability Likert-Type Scales using Cronbach's alpha coefficient. Cronbach's alpha coefficient was calculated for overall perceived barriers to maintaining body weight, as well for individual barriers set, $\mathrm{HE}$ and PA barriers. The values of Cronbach's alpha coefficient for overall perceived barriers to maintaining body weight (91 items) were 0.913 , for HE barriers (41 items) was 0.884 , and for PA barriers (50 items) was 0.837 , indicating a high level of internal consistency $[48,49]$.

\section{Ethical approval}

Ethical approval for the study (IRB approval) was obtained from the University of Maryland, College Park (No. 539976-1), and the Ministry of Health - Jeddah Health Affairs Directorate in Saudi Arabia (No A00118). Prior to the interview, each woman was asked to read and sign a consent form, which stated the purpose of the study, that participation was voluntary, and that women's responses were to be kept confidential.

\section{Statistical Analysis}

All analyses were based on the complex sampling design using the SPSS Complex Samples Software (Version 23.0). The complex sample design was accounted for in all analyses through the use of a sampling weight to account for unequal chances of selection, a sampling error stratum and sampling error computing unit (clinic), and a Taylor Series Linearization (TLS) method for variance estimation. Descriptive statistics (using the SPSS subprogram CSDESCRIPTIVES) were used to describe the characteristics of the study population and its mean, standard error, median, frequency, and percentage. Frequencies and percentages were calculated on the proportion of participants according to different variables. Multiple regression (using the SPSS subprogram CSGLM) and a binary logistic regression (using the SPSS subprogram CSLOGISTIC) were conducted to assess the associations between perceived barriers to maintaining body weight (HE and PA barriers), general obesity (BMI) and abdominal obesity (WC), respectively. Chi-square tests (using the SPSS subprogram CSTABULATE) were used to examine the statistical significance and extent of associations between barriers and socio-demographic variables. The General Linear Model (using the SPSS subprogram CSGLM) was used to assess the correlation between the perceived barriers to maintaining body weight, and EHs and PA level, as well to assess the correlation between EHs, and PA level. A p value $<.05$ was considered statistically significant. In the logistic regression analysis, the BMI levels (dependent variable) were divided into two groups: obese $\left(\mathrm{BMI} \geq 25 \mathrm{~kg} / \mathrm{m}^{2}\right)$ and non-obese $(\mathrm{BMI}<25 \mathrm{~kg} /$ $\mathrm{m}^{2}$ ). The distribution of PA level was skewed to the right, and hence the log-transformed PA levels had been used for regression analysis.

\section{Results}

Socio-demographic characteristics, obesity prevalence, eating habits (EHs), physical activity (PA) level, and lifestyle behaviors of study participants

Descriptive statistics for continuous variables are presented as mean \pm SE. The mean age (mean $\pm \mathrm{SE}$ ) of study participants was 30.27 years \pm 0.74 , and the majority (51.4\%) of the women were in the age group of $20-35$ years, $64 \%$ were married, $53.4 \%$, were housewives. Moreover, $54.2 \%$ had more than a high school diploma, and belonged to low (39.1\%) or middle (52.8\%) income levels. 
Table 1 showed that nearly $34 \%$ of women included in this study population were obese, $29.5 \%$ were overweight, $25.1 \%$ of women were abdominally obese based on WHO criteria.

\begin{tabular}{|c|c|c|c|}
\hline Anthropometric characteristics & Number & Percentage of total $^{\mathbf{a}}$ & Mean $\pm \mathbf{S E}^{\mathbf{b}}$ \\
\hline Height $(\mathrm{cm})($ mean \pm SE) & & & $156.5 \pm 0.4$ \\
\hline Weight $(\mathrm{kg})($ mean \pm SE) & & & $67.7 \pm 1.3$ \\
\hline BMI $\left(\mathbf{k g} / \mathbf{m}^{2}\right)$ WHO cutoff & & & \\
\hline Underweight $(<18.5)$ & 41 & 10.1 & \\
\hline Normal $(18.5-24.9)$ & 108 & 26.6 & \\
\hline Overweight $(25-29.9)$ & 121 & 29.5 & \\
\hline Obese $(\geq 30)$ & 138 & 33.8 & \\
\hline WC $(\mathbf{c m})($ mean \pm SE) & & & $80.4 \pm 1.1$ \\
\hline WC $(\mathbf{c m})$ WHO cutoff & & & \\
\hline Normal $(<88)$ & 306 & 75.0 & \\
\hline Health Risk $(\geq 88)$ & 102 & 25.1 & \\
\hline
\end{tabular}

WHO: World Health Organization; BMI: Body mass index; WC: waist circumference; kg: kilogram; $\mathrm{m}^{2}$ : meter square; $\mathrm{cm}$ : centimeter

aPercentage may not total to $100 \%$ due to rounding

bmean \pm standard error

Table 1: Anthropometric characteristics of non-pregnant Saudi women of reproductive age residing in Jeddah city, Saudi Arabia $(\mathrm{n}=408)$

Regarding eating habits (EHs) (Table 2), 46\% of the women had healthy EHs (satisfactory eating habits), and more than half of the women had two main meals daily. Only $26 \%$ of women consumed at least two portions (200gms) of vegetables per day, and $13 \%$ consumed at least two portions of fruit per day (200gms). Also, more than half of the women (56.2\%) drank less than the recommended amount of water daily (at least eight cups of water/day), and consumed a high amount of Western fast foods [50].

\begin{tabular}{|c|c|c|c|}
\hline Variables & $\mathbf{n}(\%)^{b}$ & Variables & $\mathbf{n}(\%)^{\mathbf{b}}$ \\
\hline Eating Habits Scores $(\text { mean } \pm S E)^{a}$ & $37.5 \pm 0.27$ & Eat while watching the TV & \\
\hline Inadequate Eating Habits & $97(24.0)$ & Always & $84(20.5)$ \\
\hline Partially Satisfactory Eating Habits & $122(30.0)$ & Often & $99(24.2)$ \\
\hline Satisfactory Eating Habits & $189(46.0)$ & Sometime & $104(25.5)$ \\
\hline Number of main meals/day $($ mean \pm SE) & $2.4 \pm .04$ & Never & $121(29.8)$ \\
\hline One & $17(4.1)$ & Fast food Consumption & \\
\hline Two & $219(53.8)$ & Yes & $345(87.7)$ \\
\hline Three & $172(42.1)$ & No & $63(15.3)$ \\
\hline Breakfast Consumption & & $\begin{array}{l}\text { Number of fast food Consumption/ } \\
\text { week }\end{array}$ & \\
\hline Always & $174(42.5)$ & One & $111(27.2)$ \\
\hline Often & $70(17.1)$ & Two or more & $234(72.8)$ \\
\hline Sometime & $121(29.6)$ & Types of foods usually consumed ${ }^{d}$ & \\
\hline Never & $43(10.6)$ & $\begin{array}{c}\text { Traditional Saudi foods (e.g., Kabsa, } \\
\text { Qursan, or Jarish) }\end{array}$ & $398(97.6)$ \\
\hline Consume (200gm) of fruit/day & & $\begin{array}{c}\text { Local fast foods (e.g., falafel, } \\
\text { Shawarma, Masoob, or Motabag) }\end{array}$ & $205(50.2)$ \\
\hline Always & $53(13.0)$ & $\begin{array}{l}\text { American fast foods (e.g., } \\
\text { McDonalds, or Burger King) }\end{array}$ & $187(46.0)$ \\
\hline Often & $82(20.0)$ & $\begin{array}{l}\text { Mediterranean foods (e.g., Lebanese, } \\
\text { or Egyptian, foods) }\end{array}$ & $157(38.5)$ \\
\hline Sometime & $225(55.3)$ & $\begin{array}{c}\text { Asian foods (e.g., Indian, Chains, or } \\
\text { Thai foods) }\end{array}$ & $32(7.8)$ \\
\hline Never & $47(11.6)$ & $\begin{array}{l}\text { Physical activity and sedentary } \\
\text { lifestyles }\end{array}$ & \\
\hline Consume (200gm) of vegetables/day & & $\begin{array}{l}\text { Waking } 150 \text { mints or more } \\
\text { /week }\end{array}$ & $118(29)$ \\
\hline Always & $105(25.9)$ & Sitting hours/day $($ mean $\pm S E)$ & $2.85 \pm 0.1$ \\
\hline Often & $144(35.2)$ & $\begin{array}{l}\text { Physical activity (MET min/week) } \\
(\text { (mean } \pm S E)\end{array}$ & $953.11 \pm 62.9$ \\
\hline
\end{tabular}




\begin{tabular}{|c|c|c|c|}
\hline Variables & $\mathbf{n}(\%)^{\mathbf{b}}$ & Variables & $\mathbf{n}(\%)^{b}$ \\
\hline Sometime & $141(34.6)$ & Low activity & $131(32.0)$ \\
\hline Never & $18(4.3)$ & Moderate activity & $262(64.2)$ \\
\hline Snacks consumption/day & & Vigorous activity & $15(3.8)$ \\
\hline Always & $92(22.6)$ & & \\
\hline Often & $115(28.2)$ & $\begin{array}{l}\text { Sleeping hours per night } \\
(\text { mean } \pm \text { SE })\end{array}$ & $6.5 \pm 0.1$ \\
\hline Sometime & $166(40.6)$ & Taking a nap & $220(54.1)$ \\
\hline Never & $35(8.6)$ & Leisure time activities ${ }^{d}$ & \\
\hline Drink 8 cups of water/day & & Walking & $18(4.3)$ \\
\hline Always & $69(16.9)$ & Sport & $8(1.9)$ \\
\hline Often & $36(8.8)$ & Shopping & $27(6.4)$ \\
\hline Sometime & $74(18.1)$ & Dancing & $24(5.8)$ \\
\hline Never & $229(56.2)$ & $\begin{array}{l}\text { Watching TV/listening to music / } \\
\text { using a computer or phone/ reading }\end{array}$ & $358(87.8)$ \\
\hline Drink a cup of milk products/day & & $\begin{array}{l}\text { Main reasons for doing regular } \\
\text { physical activities }{ }^{\mathrm{d}}\end{array}$ & \\
\hline Always & $169(41.4)$ & Health benefits & $301(73.8)$ \\
\hline Often & $74(18.3)$ & To lose weight & $194(47.6)$ \\
\hline Sometime & $114(27.8)$ & Recreation & $51(12.4)$ \\
\hline Never & $51(12.5)$ & To lose weight & $194(47.6)$ \\
\hline Eating late after $8 \mathrm{pm} /$ day & & Using cars for transportation & $404(99.2)$ \\
\hline Always & 195(47.9) & & \\
\hline Often & $100(24.5)$ & & \\
\hline Sometime & $69(16.9)$ & & \\
\hline Never & $44(10.7)$ & & \\
\hline
\end{tabular}

${ }^{a}$ mean \pm standard error

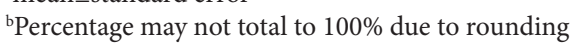

'MET:Metabolic Equivalent Task

${ }^{\mathrm{d}}$ Respondents can select more than one answer or choice (multiple response items)

Table 2: Eating habits, physical activity and sedentary lifestyles in non-pregnant Saudi women of reproductive age residing in Jeddah city, Saudi Arabia $(\mathrm{n}=408)$

Regarding the PA levels, about $64.2 \%$ of the women engaged in moderate PA, and $99.2 \%$ of them had used cars for transportation. We found $87.8 \%$ of the women spent their leisure time in sedentary behaviors (e.g., watching TV, using the phone, or using the computer), and they sat nearly 3 hours during the day $(2.85 \pm 0.1$ hours/day). Moreover, their average amount of sleep per night was $6.5 \pm 0.1$, and $54.1 \%$ of them took an afternoon nap (siesta) (Table 2).

\section{Perceived barriers to healthy eating (HE)}

According to perceived barriers to HE, a total of 201 (49.2\%) out of 408 women had barriers to HE (Table 3). The overall potential range of scores on the HE barriers scale was 0 to 123. The mean and the median of the HE score were $61.6 \pm 1.66,63.5$ score, respectively. Physical environmental barriers ranked as the important barriers to HE (53.5\%), followed by social environmental (51.1\%), and personal barriers (49.2\%). The study found that overall barriers to HE was significantly higher among adolescents (15-19 years) ( $\mathrm{p}=<.028,58.8 \%)$, but not significantly associated with education, income, marital, and occupation status (Table 4$)$.

\begin{tabular}{|c|c|c|c|c|}
\hline $\begin{array}{c}\text { Perceived barriers to maintain body } \\
\text { weigh }\end{array}$ & $\begin{array}{c}\text { Not. Imp. B. } \\
\mathbf{n}(\%)\end{array}$ & Imp. B. n(\%) & Rank-items & $\begin{array}{c}\text { Rank-sub- } \\
\text { groups }\end{array}$ \\
\hline Over all healthy eating barriers & $207(50.7)$ & $201(49.2)$ & & 3 \\
\hline a. Personal to healthy eating barriers & $207(50.8)$ & $201(49.2)$ & & $\mathrm{a}(3)$ \\
\hline Lack of Willpower & $193(47.4)$ & $215(52.6)$ & 5 & $\mathrm{a}(4)$ \\
\hline Lack of Knowledge & $197(48.2)$ & $211(51.8)$ & 7 & $\mathrm{a}(2)$ \\
\hline Lack of Skills & $173(42.3)$ & $235(57.7)$ & 2 & $\mathrm{a}(1)$ \\
\hline Lack of Enjoyment & $169(41.5)$ & $239(58.5)$ & & 2 \\
\hline b. Social environmental to healthy & $199(48.9)$ & $209(51.1)$ & & $\mathrm{b}(1)$ \\
\hline
\end{tabular}




\begin{tabular}{|c|c|c|c|c|}
\hline $\begin{array}{c}\text { Perceived barriers to maintain body } \\
\text { weigh }\end{array}$ & $\begin{array}{l}\text { Not. Imp. B. } \\
\mathbf{n}(\%)\end{array}$ & Imp. B. $\mathbf{n}(\%)$ & Rank-items & $\begin{array}{l}\text { Rank-sub- } \\
\text { groups }\end{array}$ \\
\hline Social Influence & $196(48.2)$ & $212(51.8)$ & 6 & $\mathrm{~b}(2)$ \\
\hline Lifestyle changes & $151(53.7)$ & $257(46.3)$ & 8 & $\mathrm{~b}(3)$ \\
\hline $\begin{array}{l}\text { c. Physical environmental to healthy } \\
\text { eating barriers }\end{array}$ & $190(46.5)$ & $218(53.5)$ & & 1 \\
\hline Lack of resources & $190(46.5)$ & $218(53.5)$ & 4 & $c(1)$ \\
\hline Overall physical activity barriers & 201(49.3) & $207(50.7)$ & & \\
\hline a. Personal barriers to physical activity & $210(51.9)$ & 198(48.6) & & 3 \\
\hline Lack of Willpower & $163(40.0)$ & $245(60.0)$ & 2 & $\mathrm{a}(1)$ \\
\hline Lack of self-confidence & $229(56.2)$ & $179(43.8)$ & 12 & $a(6)$ \\
\hline Lack of Skills & $202(49.5)$ & $206(50.5)$ & 11 & $a(5)$ \\
\hline Lack of Enjoyment & $269(65.9)$ & $139(34.1)$ & 14 & $\mathrm{a}(8)$ \\
\hline Lack of Knowledge & $174(42.7)$ & $234(57.3)$ & 5 & $\mathrm{a}(2)$ \\
\hline Lack of Energy & 193(47.3) & $215(52.7)$ & 10 & $\mathrm{a}(4)$ \\
\hline Fear of Injury & $180(44.1)$ & $228(55.9)$ & 7 & $a(3)$ \\
\hline Health Problems & $200(62.0)$ & $155(38.0)$ & 13 & $\mathrm{a}(7)$ \\
\hline $\begin{array}{l}\text { b. Social environmental to physical } \\
\text { activity barriers }\end{array}$ & $205(51.1)$ & $203(49.9)$ & & 2 \\
\hline Lack of Support & $173(42.9)$ & $235(57.6)$ & 4 & $\mathrm{~b}(2)$ \\
\hline Lack of Time & $149(36.4)$ & $259(63.6)$ & 1 & $\mathrm{~b}(1)$ \\
\hline Social Influence & $181(44.3)$ & $227(55.7)$ & 8 & $\mathrm{~b}(4)$ \\
\hline Social Norms & $176(43.2)$ & $232(56.8)$ & 6 & $\mathrm{~b}(3)$ \\
\hline $\begin{array}{l}\text { c. Physical environmental to physical } \\
\text { activity barriers }\end{array}$ & $177(43.3)$ & $231(56.6)$ & & 1 \\
\hline Lack of resources & 191(46.7) & $217(53.3)$ & 9 & $c(2)$ \\
\hline Lack of Transportation & $193(47.3)$ & $215(52.7)$ & 10 & $c(3)$ \\
\hline Hot Weather & $164(40.1)$ & $244(59.9)$ & 3 & $c(1)$ \\
\hline
\end{tabular}

Imp. B.: important barrier; Not. Imp. B.: not important barriers

"Barriers were rated on 4-point Likert scale that ranged from "very likely" (3) to "very unlikely" (0)

The median score for the scale was used to divide barriers score into important versus not important barriers

Table 3: Perceived barriers ${ }^{a}$ scoring to healthy eating habits and physical activity among non-pregnant Saudi women

of reproduction age residing in Jeddah city, Saudi Arabia $(n=408)$

Regarding personal barriers to the HE subgroups score, the lack of enjoyment (58.5\%) was found to be the most important personal barriers to HE reported by women, followed by lack of skills (57.7\%), willpower (52.6\%), and knowledge (51.8\%) (Table 3). The analysis revealed that personal barriers to HE was significantly higher in adolescents (15-19 years) $(\mathrm{p}=<.001,69.7 \%)$, and in the women with low-income levels $(\mathrm{p}=<.011,62.9 \%)$, and never married women $(\mathrm{p}=<.049,60.9 \%)$, but was not significantly associated with education and occupation status (Table 4).

\begin{tabular}{|c|c|c|c|c|c|c|c|c|c|c|c|c|}
\hline \multirow[b]{2}{*}{ Variables } & \multicolumn{3}{|c|}{ Over all HE barriers } & \multicolumn{3}{|c|}{$\begin{array}{l}\text { Personal } \\
\text { HE barriers }\end{array}$} & \multicolumn{3}{|c|}{$\begin{array}{c}\text { Social environmental } \\
\text { HE barriers }\end{array}$} & \multicolumn{3}{|c|}{$\begin{array}{c}\text { Physical environmental } \\
\text { HE barriers }\end{array}$} \\
\hline & $\begin{array}{l}\text { Imp. } \\
\text { Barriers }\end{array}$ & $\begin{array}{l}\text { Not. Imp } \\
\text { Barriers }\end{array}$ & Pvalue ${ }^{\mathrm{b}}$ & $\begin{array}{l}\text { Imp. } \\
\text { Barriers }\end{array}$ & $\begin{array}{l}\text { Not. Imp. } \\
\text { Barriers }\end{array}$ & P value ${ }^{b}$ & $\begin{array}{l}\text { Imp. } \\
\text { Barriers }\end{array}$ & $\begin{array}{l}\text { Not. Imp. } \\
\text { Barriers }\end{array}$ & Pvalue ${ }^{b}$ & $\begin{array}{l}\text { Imp. } \\
\text { Barriers }\end{array}$ & $\begin{array}{l}\text { Not. Imp. } \\
\text { Barriers }\end{array}$ & Pvalue $^{\mathrm{b}}$ \\
\hline Age groups & & & $.028^{*}$ & & & $.001^{*}$ & & & .671 & & & .572 \\
\hline Adolescents (15-19yrs) & $41(58.8)$ & $29(41.2)$ & & $49(69.7)$ & $21(30.3)$ & & $34(48.8)$ & $36(51.2)$ & & $41(58.5)$ & $29(41.5)$ & \\
\hline Young women (20-35yrs) & $112(53.3)$ & $98(46.7)$ & & $106(50.7)$ & $103(49.3)$ & & $113(54.0)$ & $96(46.0)$ & & $109(52.0)$ & $101(48.0)$ & \\
\hline Middle age (36-49yrs) & $48(7.9)$ & $80(62.5)$ & & $45(35.3)$ & $83(64.7)$ & & $61(47.9)$ & $76(52.1)$ & & $68(53.3)$ & $60(46.7)$ & \\
\hline Education level & & & .160 & & & .193 & & & .086 & & & .061 \\
\hline Less than high school & $59(53.1)$ & $67(46.9)$ & & $66(52.8)$ & $59(47.2)$ & & $53(47.3)$ & $72(57.7)$ & & $73(58.4)$ & $52(41.6)$ & \\
\hline Completed high school & $57(58.1)$ & $41(49.9)$ & & $56(56.9)$ & $42(43.1)$ & & $58(58.8)$ & $41(41.2)$ & & $59(60.4)$ & $39(39.9)$ & \\
\hline More than high school & $85(46.3)$ & $99(53.7)$ & & $79(56.9)$ & $106(57.3)$ & & $98(53.2)$ & $86(46.8)$ & & $86(53.3)$ & $98(46.7)$ & \\
\hline Income level & & & .227 & & & $.011^{*}$ & & & .094 & & & .178 \\
\hline Low income $(<8,000 \mathrm{SAR})$ & $74(46.2)$ & $86(53.8)$ & & $100(62.9)$ & $59(37.1)$ & & $74(46.7)$ & $85(53.3)$ & & $97(61.1)$ & $62(38.9)$ & \\
\hline $\begin{array}{l}\text { Middle income } \\
(8000-18,000 \mathrm{SAR})\end{array}$ & $65(48.6)$ & $111(51.4)$ & & $93(43.0)$ & $123(57.0)$ & & $123(57.0)$ & $93(43.0)$ & & $106(49.1)$ & $110(50.9)$ & \\
\hline
\end{tabular}




\begin{tabular}{|c|c|c|c|c|c|c|c|c|c|c|c|c|}
\hline \multirow[b]{2}{*}{$\begin{array}{l}\text { High income } \\
(>18,000 \mathrm{SAR})\end{array}$} & \multicolumn{3}{|c|}{ Over all HE barriers } & \multicolumn{3}{|c|}{$\begin{array}{c}\text { Personal } \\
\text { HE barriers }\end{array}$} & \multicolumn{3}{|c|}{$\begin{array}{c}\text { Social environmental } \\
\text { HE barriers }\end{array}$} & \multicolumn{3}{|c|}{$\begin{array}{c}\text { Physical environmental } \\
\text { HE barriers }\end{array}$} \\
\hline & $11(32.7)$ & $22(67.3)$ & & $8(23.4)$ & $25(76.6)$ & & $12(34.9)$ & $22(65.1)$ & & $15(46.1)$ & $18(53.9)$ & \\
\hline Marital status & & & .136 & & & $.049^{*}$ & & & .711 & & & .590 \\
\hline Never married & $72(56.5)$ & $55(43.5)$ & & $77(60.9)$ & $50(39.1)$ & & $63(49.8)$ & $64(50.2)$ & & $71(56.2)$ & $56(48.0)$ & \\
\hline Married & $129(46.1)$ & $152(53.9)$ & & $123(43.9)$ & $158(56.1)$ & & $146(48.2)$ & $135(51.8)$ & & $147(52.3)$ & $134(47.7)$ & \\
\hline Occupation status & & & .175 & & & .369 & & & .803 & & & .838 \\
\hline Housewife & $116(57.8)$ & $102(49.1)$ & & $115(52.8)$ & $103(47.2)$ & & $113(51.8)$ & $105(48.2)$ & & $120(55.0)$ & $98(51.8)$ & \\
\hline Employed & $41(47.4)$ & $46(52.6)$ & & $42(48.2)$ & $45(51.8)$ & & $47(53.7)$ & $40(46.6)$ & & $45(52.1)$ & $42(47.9)$ & \\
\hline Student & $44(42.3)$ & $59(57.7)$ & & $44(42.4)$ & $59(57.6)$ & & $49(47.8)$ & $54(52.2)$ & & $53(51.8)$ & $50(48.2)$ & \\
\hline
\end{tabular}

Imp. B.: important barrier; Not. Imp. B.: not important barriers.

"Barriers were rated on 4-point Likert scale that ranged from "very likely" (3) to "very unlikely" (0)

The median score for the scale was used to divide barriers score into important versus not important barriers.

${ }^{\mathrm{b}} \mathrm{chi}$-square test of independence,

* significant $\mathrm{p}=<.05$

Table 4: Socio-demographic characteristics by perceived barriers ${ }^{\mathrm{a}}$ to healthy eating (HE) among non-pregnant Saudi

women of reproduction age residing in Jeddah city, Saudi Arabia $(n=408)$

According to social environmental barriers to HE subgroups score, the lack of time (61.7\%), was found to be the most important social environmental barriers to HE reported by women, followed by social influence (51.8\%), and lifestyle changes (46.3\%) (Table 3 ). The analysis indicated that the overall social environmental barriers score to HE were not significantly associated with all social-demographic variables (age, education, income, marital, and occupation status) (Table 4).

Regarding physical environmental barriers to HE subgroup score, the lack of resources (53.5\%) was found to be the most important barriers to HE reported by women (Table 4). The results indicated that overall physical environmental barriers score to adapting healthy eating habits were not significantly associated with all social-demographic variables (age, education, income, marital, and occupation status) (Table 5).

\begin{tabular}{|c|c|c|c|c|c|c|c|c|c|c|c|c|}
\hline \multirow[b]{2}{*}{ Variables } & \multicolumn{3}{|c|}{ Over all PA barriers } & \multicolumn{3}{|c|}{$\begin{array}{l}\text { Personal } \\
\text { PA barriers }\end{array}$} & \multicolumn{3}{|c|}{$\begin{array}{l}\text { Social environmental } \\
\text { PA barriers }\end{array}$} & \multicolumn{3}{|c|}{$\begin{array}{c}\text { Physical environmental } \\
\text { PA barriers }\end{array}$} \\
\hline & $\begin{array}{l}\text { Imp. } \\
\text { Barriers }\end{array}$ & $\begin{array}{l}\text { Not. Imp } \\
\text { Barriers }\end{array}$ & Pvalue $^{\mathrm{b}}$ & $\begin{array}{l}\text { Imp. } \\
\text { Barriers }\end{array}$ & $\begin{array}{l}\text { Not. Imp. } \\
\text { Barriers }\end{array}$ & P value $^{\mathrm{b}}$ & $\begin{array}{l}\text { Imp. } \\
\text { Barriers }\end{array}$ & $\begin{array}{l}\text { Not. Imp. } \\
\text { Barriers }\end{array}$ & Pvalue ${ }^{b}$ & $\begin{array}{l}\text { Imp. } \\
\text { Barriers }\end{array}$ & $\begin{array}{l}\text { Not. Imp. } \\
\text { Barriers }\end{array}$ & Pvalue $^{\mathrm{b}}$ \\
\hline Age groups & & & .188 & & & .083 & & & $.024^{*}$ & & & $.040^{*}$ \\
\hline Adolescents (15-19yrs) & $44(62.6)$ & $26(37.4)$ & & $44(62.5)$ & $26(37.5)$ & & $46(64.8)$ & $25(35.5)$ & & $41(58.3)$ & $29(47.7)$ & \\
\hline Young women (20-35yrs) & $106(50.6)$ & $104(49.4)$ & & $92(43.9)$ & $118(56.1)$ & & $111(53.0)$ & $99(47)$ & & $131(62.6)$ & $78(37.4)$ & \\
\hline Middle age (36-49yrs) & $57(44.2)$ & $71(55.8)$ & & $62(48.5)$ & $66(51.5)$ & & $51(59.7)$ & $76(40.0)$ & & $58(45.7)$ & $69(54.3)$ & \\
\hline Education level & & & .106 & & & $.001^{*}$ & & & .427 & & & .514 \\
\hline Less than high school & $75(59.9)$ & $50(40.1)$ & & $79(63.3)$ & $46(36.7)$ & & $67(53.6)$ & $58(46.4)$ & & $66(52.4)$ & $60(47.6)$ & \\
\hline Completed high school & $47(52.2)$ & $51(47.8)$ & & $48(48.9)$ & $50(51.1)$ & & $54(54.8)$ & $44(45.2)$ & & $55(55.6)$ & $44(44.4)$ & \\
\hline More than high school & $85(54.0)$ & $100(46.0)$ & & $71(38.3)$ & $114(61.7)$ & & $87(47.2)$ & $97(52.8)$ & & $111(60.0)$ & $74(40.1)$ & \\
\hline Income level & & & $.025^{*}$ & & & $.027^{\star}$ & & & .067 & & & .551 \\
\hline $\begin{array}{l}\text { Middle income } \\
\text { (8000-17,999SAR) }\end{array}$ & $99(46.0)$ & $116(54.0)$ & & 95(55.9) & $120(44.1)$ & & $98(45.3)$ & $118(54.7)$ & & $118(54.6)$ & $98(45.4)$ & \\
\hline $\begin{array}{l}\text { High income } \\
(>18,000 \mathrm{SAR})\end{array}$ & $12(36.3)$ & $21(63.7)$ & & $9(27.3)$ & $24(72.7$ & & $17(51.8)$ & $16(48.2)$ & & $17(512)$ & $16(48.8)$ & \\
\hline Marital status & & & .074 & & & .782 & & & .396 & & & .078 \\
\hline Never married & $72(56.6)$ & $55(43.4)$ & & $63(49.6)$ & $64(50.4)$ & & $68(53.9)$ & $59(46.1)$ & & $79(62.4)$ & $48(37.6)$ & \\
\hline Married & $135(48.0)$ & $146(52.0)$ & & $135(48.1)$ & $146(51.9)$ & & $140(49.7)$ & $141(50.3)$ & & $152(54.0)$ & $129(46.0)$ & \\
\hline Occupation status & & & .383 & & & .237 & & & .107 & & & .344 \\
\hline Housewife & $117(53.8)$ & $101(46.2)$ & & $114(52.3)$ & 104(47.7) & & $120(55.3)$ & $97(44.7)$ & & $125(57.4)$ & $93(42.4)$ & \\
\hline Employed & $44(50.1)$ & $44(49.9 \%)$ & & $42(47.9)$ & $45(52.1)$ & & $43(49.6)$ & $44(50.4)$ & & $54(62.1)$ & $33(37.9)$ & \\
\hline Student & $46(44.6)$ & $57(55.4)$ & & $43(41.3)$ & $60(58.7)$ & & $45(43.2)$ & $58(56.8)$ & & $52(50.2)$ & $51(49.8)$ & \\
\hline
\end{tabular}

Imp. B.: important barrier; Not. Imp. B.: not important barriers.

"Barriers were rated on 4-point Likert scale that ranged from "very likely" (3) to "very unlikely" (0)

The median score for the scale was used to divide barriers score into important versus not important barriers.

${ }^{b}$ chi-square test of independence,

*significant $\mathrm{p}=<.05$

Table 5: Socio-demographic characteristics by perceived barriers ${ }^{\mathrm{a}}$ to physical activity (PA) among non-pregnant

Saudi women of reproduction age residing in Jeddah city, Saudi Arabia $(\mathrm{n}=408)$ 


\section{Perceived barriers to physical activity (PA)}

According to perceived barriers to PA, a total of 207 (50.7\%) out of 408 women had barriers to engaging in PA. The overall potential range of scores on the PA barriers scale was 0 to 147 . The mean and the median of the PA barriers score were 76.4 \pm 1.5 , 78.0 score, respectively. Physical environmental barriers to PA ranked as the most important barriers to PA (56.6\%), followed by social environmental (51.6\%), and personal barriers (48.6\%) (Table 3). The analysis revealed that overall barriers to engaged PA score was significantly higher among women in low-income level $(\mathrm{p}=<.025,59.9 \%)$, but not significantly associated with age, education, marital, and occupation status (Table 5).

Regarding personal barriers to PA subgroups score, lack of willpower (60.0\%), was found to be the most important personal barriers to PA reported by women, followed by lack of knowledge (57.3\%), fear of injury (55.9\%), lack of energy (52.7\%), lack of skills $(50.5 \%)$, lack of self-confidence (43.8\%), having healthy problems (38\%), and lack of enjoyment (34.1\%) (Table 3). The personal PA barriers score was significantly higher among women with less than a high school diploma $(\mathrm{p}=<.001,63.3 \%)$, and was significantly higher among women in low-income levels $(\mathrm{p}=<.027,58.9 \%)$, but not significantly associated with age, marital, and occupation status (Table 5).

According to social environmental barriers to PA subgroups score, the lack of time (63.6\%) was found to be the most important social environmental barriers to PA reported by women, followed by lack of support (57.6\%), social norms (56.6\%), and social influence (55.7\%) (Table 2). Social environmental PA barriers score was significantly higher among adolescents (15-19 years) $(\mathrm{p}=<.024,64.8 \%)$, but was not significantly associated with education level, income, marital, and occupation status (Table 5).

Regarding the physical environmental PA barriers subgroups score, the hot weather (59.9\%) was found to be the most important physical environmental barrier to PA reported by women, followed by lack of resources (53.3\%), and lack of transportation (52.7\%) (Table 3). Physical environmental PA barriers score was significantly higher among women in age group (20-35 years) ( $\mathrm{p}=<.040$, 62.6\%), but was not significantly associated with education level, income, marital, and occupation status (Table 5).

\section{Relationship between total perceived barriers scores to maintaining healthy body weight (HEs and PA barriers) and eating habits (EHs) and PA levels (PA)}

The maximum possible score of perceived barriers to HE was 123 , with a mean of $61.6 \pm 1.6$, and a median of 63.5 score. While the maximum possible score of EHs was 56 with mean of $37.5 \pm 0.27$, the results show a significant negative correlation between EHs score and the total score of perceived barriers to $\mathrm{HE}(\mathrm{p}=<.001, \mathrm{r}=-0.26)$ among the study population. In addition, there was a significant negative correlation between EHs, personal $(\mathrm{p}=<.001, \mathrm{r}=-0.3)$ and the social environmental barriers $(\mathrm{p}=<.026, \mathrm{r}=-0.2)$ to $\mathrm{HE}$, while there was no correlation with the physical environmental barriers to $\mathrm{HE}(\mathrm{p}=<.676, \mathrm{r}=-0.03)$.

\begin{tabular}{|c|c|c|}
\hline & \multicolumn{2}{|c|}{ PA (MET min/week) ${ }^{a}$} \\
\hline & $\mathbf{r}^{\mathbf{b}}$ & $P$ value \\
\hline \multirow[t]{3}{*}{ EHs score } & 0.16 & $.001^{*}$ \\
\hline & \multicolumn{2}{|c|}{ Overall PA barrier score } \\
\hline & $\mathbf{r}^{\mathrm{c}}$ & $P$ value \\
\hline \multirow[t]{3}{*}{ Overall HE barrier score } & 0.62 & $<.001^{\star}$ \\
\hline & \multicolumn{2}{|c|}{ EHs score } \\
\hline & $\mathbf{r}^{c}$ & $P$ value \\
\hline Overall HE barrier score & -0.26 & $<.001^{*}$ \\
\hline Personal HE barrier & -0.3 & $<.001^{*}$ \\
\hline Social environmental HE barrier & -0.2 & $.026^{*}$ \\
\hline \multirow[t]{3}{*}{$\begin{array}{c}\text { Physical environmental HE } \\
\text { barrier }\end{array}$} & 0.03 & .676 \\
\hline & \multicolumn{2}{|c|}{ PA (MET min/week) ${ }^{a}$} \\
\hline & $\mathbf{r}^{\mathrm{c}}$ & P value \\
\hline Overall PA barrier score & 0.06 & .564 \\
\hline Personal PA barrier & 0.05 & .537 \\
\hline Social environmental PA barrier & 0.5 & .127 \\
\hline $\begin{array}{c}\text { Physical environmental PA } \\
\text { barrier }\end{array}$ & 0.08 & .143 \\
\hline
\end{tabular}

MET: Metabolic Equivalent Task; HE: healthy eating; PA: physical activity; EHs: eating habits.

a logarithm of PA,

b Spearman correlation,

' Pearson Correlation.

* Correlation is significant at the 0.05 level (2-tailed)

Table 6: Correlation between perceived barriers to maintaining body weight (healthy eating and physical activity barriers) and eating habits score and physical activity level among non-pregnant Saudi women of reproductive age residing in Jeddah city, Saudi Arabia ( $\mathrm{n}=408$ ) 
The maximum possible score of perceived barriers to PA was 150 score with a mean of $76.4 \pm 1.5$ and a median of 78.0 score, while the mean for PA level was 953.1 \pm 63.0 (MET min/week). The results show no significant correlation was found between PA level and the total score of perceived barriers to PA $(\mathrm{p}=<.546, \mathrm{r}=0.06)$ among the study population, or with the score of PA barriers subgroups (personal, social, and environmental barriers). Moreover, the results show a significant positive correlation between perceived barriers to HE and perceived barriers to $\mathrm{PA}(\mathrm{p}=<.001, \mathrm{r}=0.62)$, as well a significant positive correlation between EHs and PA levels $(\mathrm{p}=<.001, \mathrm{r}=0.16)$ (Table 6).

\section{Logistic regression analysis and obesity (BMI) and perceived barriers to maintaining healthy body weight}

A binary logistic regression was run to test the predictive relationship between the perceived barriers that Saudi women face in HE and PA and general obesity $\left(\mathrm{BMI} \geq 25 \mathrm{~kg} / \mathrm{m}^{2}\right)$ (Table 7$)$. The overall regression model was not significant, and explained $1.5 \%(\mathrm{Cox}$ $\&$ Snell) and 1.2\% (Nagelkerke) of the variance in obesity, Wald $F(2)=5.22, p=.074$. The perceived barriers to PA was a significant predictor within the model $(\mathrm{p}=.024)$, but not the perceived barriers of $\mathrm{HE}(\mathrm{p}=.183)$.

\begin{tabular}{|c|c|c|c|c|c|c|}
\hline Barriers & B & SE & Wald $x^{2}$ & $P$ value & Odds ratio & $95 \% \mathrm{CI}$ \\
\hline $\begin{array}{c}\text { Perceived barriers of healthy } \\
\text { eating }\end{array}$ & 0.02 & 0.02 & 1.80 & 0.18 & 1.02 & $\begin{array}{l}0.97- \\
1.70\end{array}$ \\
\hline $\begin{array}{c}\text { Perceived barriers to being } \\
\text { physically activity }\end{array}$ & 0.19 & 0.01 & 5.10 & .02 & 0.98 & $\begin{array}{c}0.96- \\
1.00\end{array}$ \\
\hline Constant & -0.38 & 0.60 & 0.53 & .531 & 0.70 & $\begin{array}{l}0.18- \\
2.62\end{array}$ \\
\hline \multicolumn{7}{|c|}{ Wald $x^{2} 5.22(d f=2), P$ value $=.074$} \\
\hline Cox and Snell pseudo $\mathrm{R}^{2}$ & 0.051 & & & & & \\
\hline Nagelkerke pseudo $\mathbf{R}^{2}$ & 0.021 & & & & & \\
\hline \multicolumn{7}{|c|}{$\begin{array}{l}\text { B: Coefficient; SE: stander error; CI: confidence interval } \\
\text { Table7: Logistic regression of association between general obesity (BMI) and the perceived barriers to } \\
\text { maintaining body weight in non-pregnant Saudi women of reproduction age residing in Jeddah city } \\
\text { Saudi Arabia }(n=408)\end{array}$} \\
\hline
\end{tabular}

\section{Multiple linear regression analysis and abdominal obesity and perceived barriers to maintaining healthy body weight}

A multiple linear regression was run to test the predictive relationship between the perceived barriers that Saudi women face in healthy eating and being physically active and abdominal obesity (WC). The overall regression model was not significant, and explained $0.6 \%, \mathrm{R}^{2}=.006$, Wald $\mathrm{F}(2)=0.994, \mathrm{p}=.608$. Neither of the predictor variables (HE and PA barriers) were significant within the model $(\mathrm{p}=.319, \mathrm{p}=.455$, respectively) (Table 8$)$.

\begin{tabular}{|c|c|c|c|c|}
\hline Risk factors ${ }^{a}$ & B $(95 \% C I)$ & SE & Wald $x^{2}$ & $P$ value \\
\hline Constant & $80.2(71.2,89.1)$ & 4.0 & 400.6 & $<.001$ \\
\hline $\begin{array}{l}\text { Perceived barriers of healthy } \\
\text { eating }\end{array}$ & $0.074(-0.1,0.2)$ & 0.07 & 0.99 & .319 \\
\hline $\begin{array}{c}\text { Perceived barriers to } \\
\text { physical activity }\end{array}$ & $-0.09(-0.4,0.2)$ & 0.12 & 0.58 & .445 \\
\hline \multicolumn{5}{|c|}{ Wald $x^{2}: 0.99(d f=2), P$ value $=.608$} \\
\hline \multicolumn{5}{|c|}{$R^{2}: 0.006$} \\
\hline
\end{tabular}

WC: waist circumference B: Coefficient; SE: stander error; CI: confidence interval a Only significant factors are presented

Table 8: Multiple linear regressions of association between Abdominal obesity (WC) and the perceived barriers to maintaining body weight in non-pregnant Saudi women of reproduction age residing in Jeddah city, Saudi Arabia $(n=408)$

\section{Discussion}

The reproductive years are an important life stage for women; it may result in significant weight gain that could affect women's short- and long-term health and the health of their children [6]. In order to develop appropriate and effective obesity prevention strategies for Saudi women, the key point is to understand the barriers they perceive in attempting to maintaining a healthy weight. To the best of the authors' knowledge, this is the first study conducted to identify how conservative Saudi cultures, and politics affect the EHs and PA among a representative sample of non-pregnant Saudi women of reproductive age (15-49 years) obtaining services at PHCCs in Jeddah.

This study produced some important findings among the study population: (1) it estimated a high prevalence of overweight (29.5\%), obesity (33.8\%), abdominal obesity (25\%), physical inactivity (31.2\%), sedentary behaviors (87.8\%), and unhealthy EHs (24\%); (2) it identified considerable perceived personal, social, and physical environmental barriers to HE and PA; (3) it recognized some 
associations between perceived barriers to maintain healthy body weight (HE and PA barriers) and different socio-demographic characteristics; (4) it found a significant association between overall HE barriers score and age and between overall PA barriers score and income level; and (5) it illustrated a positive relationship between EHs and PA level, as well as a negative relationship between EHs score and overall HE barriers score, and HE barriers categories-personal, and social environmental barriers.

\section{Perceived barriers to maintaining a healthy body weight}

Maintaining a healthy body weight is important for overall health and can help prevent and control many diseases and conditions [4]. Previous studies in Saudi Arabia and Arab countries have concluded that Arab women face more perceived barriers to healthy lifestyle than men, particularly for PA [39,51-54]. The findings of the current study illustrate that a high proportion of Saudi women face great barriers to maintaining their healthy weight related to adapting healthy EHs or engaging in PA. Overall, nearly half of the women had high scores in HE and PA barriers, which indicated that both barriers played important roles in being unable to maintaining their body weight and having unhealthy lifestyles. These findings confirmed and were consistent with the high prevalence of overweight, obesity, physical inactivity, and unhealthy EHs among the study population. The sum score for each barrier category (personal, social, and environmental barriers) showed that nearly half or more of the women had high-score barriers for HE and PA across all barrier categories. However, the women tended to rate physical environmental barriers as the key perceived barriers to HE and PA, followed by social environmental then personal barriers. These findings are in line with those in the literature, in which there was a consensus that the physical environmental barriers are more likely to be an important source of influence on obesity-related behaviors [55]. Therefore, these findings highlight that the effort to prevent obesity should not ignore the physical environmental barriers related to the lack of recourses, facilities, and the cost to HE and PA among women who are at a high-risk of weight gain.

\section{Eating habits and perceived barriers to healthy eating}

Diet and nutrition play important roles in maintaining health and preventing numerous diseases [4]. A decrease in morbidity and mortality associated with lifestyle health diseases may be achievable if healthy EHs are adopted early in life and maintained in the long term [8]. However, the present study showed a high proportion of the women (54\%) had unhealthy EHs and (30\%) partially satisfactory EHs. Some unhealthy EHs common among the study population included (1) consuming less amounts of fruits and vegetables, (2) drinking less than the recommended amount of water daily, and (3) a high prevalence of fast-food consumption. This finding indicates that there is a need to improve those women's eating habits and for intervention to promote healthy dietary habits to reduce the prevalence of obesity.

Nearly half of the women (49.3\%) had personal barriers to HE. Moreover, the sum score of the personal barriers subgroups to HE showed that the lack of personal enjoyment to healthy foods, lack of personal skills to plan, shop for, prepare, or cook healthy foods, the lack of personal willpower, and knowledge were the most important personal barriers to HE among the study population. These findings are in line with those reported by previous studies [52,56]. Al-Farwan (2011) found that the most important personal barriers among 302 Saudi women (overweight and obese), age 16-60 years old, were the lack of personal information on healthy foods combined with the lack of skills to prepare and cook healthy foods (61.2\%) and the lack of personal motivation to eat healthy foods (56.6\%) [56]. Similar findings were observed among 327 young Kuwaiti women (age 19-26), the lack of personal skills (70\%), enjoyment (59.3\%), motivation (57.2\%), and knowledge (56.3\%) seemed to be the most important barriers to eat healthy foods among those young women [52].

Personal barriers to HE were significantly higher among adolescents, low-income, and women who had never married. The personal barriers were also seen as barriers to HE in adolescents (2240 males and 2458 females), age 15-18, in seven Arab countries (Algeria, Jordan, Kuwait, Libya, Palestine, Syria, and the United Arab Emirates) [53]. In the present study, the personal barriers to HE were significantly higher among low-income women and women who had never married and could be due to the fact that $60.1 \%(n=75)$ of the low-income women and $37.3 \%(n=47)$ of the women who had never married were less educated (less than a high school diploma) and were more likely to not have enough knowledge in nutrition and healthy foods. These findings highlight the central role of cognitive factors as barriers to healthy eating, and there is a need to improve those women's knowledge of nutrition and their skills to plan and prepare tasty healthy foods. Therefore, it is important to promote healthy eating as well as a healthy lifestyle among those women practically in the adolescent age group, and in low-income and never-married women.

Our study indicated a high prevalence of social environmental barriers to HE (51.1\%) among the study population. The sum score of the social environmental barriers subgroups to HE (lack of time, social influence, and lifestyle changes) showed that the lack of time to prepare and plan healthier meals (due to family commitments and social activities) was the most important social environmental barriers to HE among the study population. In previous studies, lack of time was one of the most frequently reported barriers in developed and developing countries [39,52,57,58]. In the current study, when the response categories very likely and somewhat likely were combined, the lack of time related to family and social commitment (reported by $39.5 \%$ ) was more common than the lack of time due to job demands $(34.1 \%)$ in the study population. The women's lack of time for HE could be related to the fact that $46.6 \%(n=190)$ of the study sample were students and employees, with $71 \%(n=73)$ of the students being married and $41 \%(n=42)$ of them having four or more children and with $65.6 \%(n=57)$ of the employed women being married and $27.5 \%(n=24)$ of the them having four or more children. Therefore, it seems difficult for them to find time to plan and cook healthy foods, and it seems that they need time-management help. 
Additionally, social relationships and interactions can have positive and negative influences on lifestyle and weight status [59]. The current study also revealed that there was a high social influence on the women's EHs (51.8\%); 56.5\% of the women reported that "usually healthy foods are not served at social activities," and $43.6 \%$ of the women reported that "it can be hard to stick with an HE plan when family and friends do not want to join" them. Therefore, the weight management interventions should include strategies that solicit support to help women lose and maintain their weight and overcome social influences and interactions.

The results revealed that more than half of the women had a lack of resources barrier (e.g., lack of money, food availability, and cooking facilities) that related to the HE physical environmental barriers. Among the lack of recourses barriers, "there's not much choice of healthy foods when eating outside of the home" was the main barrier reported by the women related to HE physical environmental barriers. This finding was consistent with a previous Saudi study that demonstrated the lack of recourses to HE was stated by $60.2 \%$ of the study group (144 men and 306 women), age $15-80$, and was significantly higher among the younger age group (15-30 years) (71.7\%) and the never-married group (91.9\%) [39]. However, no significant associations were found between HE physical environmental barriers across all socio-demographic variables among the current study's population. The lack of associations found between socio-demographic variables and physical environmental barriers to HE could be because the women in the current study belonged to a young age group, the same social class, and the majority of them were married.

\section{Physical activity (PA) level and its perceived barriers}

PA is an important component of a healthy lifestyle and has been described as an essential factor in managing many health conditions and to combating the obesity epidemic [11,12]. Using the IPAQ short-form instrument, this study showed that physical inactivity among the study sample was $31.2 \%$. These findings confirmed and were consistent with the high prevalence of physical inactivity (assessed by the IPAQ) among Saudi women reported by Al-Hazzaa (2007) [14]. Al-Hazzaa (2007) found that the physical inactivity prevalence among 362 Saudi women (age 15-75) was 34.3\%. In the Al-Hazzaa (2007) study and our study, the portion of the women walked for 150 minutes or more per week had only met (28.5\% vs $29 \%$ ) from the current USDA PA guidelines for adults, which recommend a minimum of minutes/week of moderate activity for weight management [13]. Low levels of walking among the current study population may contribute to a high dependency of the women on using cars for daily commuting, which may have limited their movements.

Consistent with other studies, the current study found that a high proportion of women faced great barriers to PA $[5,39,53,54]$. The sum scores in the current study showed that the women had high score barriers across all personal barriers subgroups for PA. The lack of willpower (60\%) was ranked as the most important personal barrier to PA, and the lack of enjoyment was ranked as less important (34.1\%). The Al-Quaiz (2009) study found that the personal barrier to PA was higher among women than men; a lack of willpower (78.9\%) was ranked as the most important personal barrier to PA among women, followed by a lack of energy (77\%), lack of skills (48.9\%), and fear of injury (22.9\%) [39]. Furthermore, in the present study, the personal barrier to PA was significantly higher among less-educated and low-income women. These findings are parallel with the high physical inactivity levels observed among those women. Nearly $38 \%$ of those with less education and $34 \%$ of those with a low-income level were physically inactive. Previous studies have concluded that a lower social class background serves as a barrier to engage in any PA or sport $[60,61]$. These findings suggest that community-based interventions and public health strategies should concentrate efforts on these subgroups in order to reach and help them overcome such barriers and increase their PA level [62].

Our study indicated a high proportion of the women had high scores in social environmental barriers to PA (49.9\%). The sum score of social environmental barriers subgroups to PA (lack of support, lack of time, social influence, and social norms barriers) showed that the lack of time engaged in PA was the most important subgroup barriers for PA social environmental barriers among the study population. These findings were in agreement with previous studies [52,57,59]. However, in our study, the other social environmental subgroups barriers that also had a considerable impact on the women's PA included the lack of support from family and friends and a lack of social influence and norms. The analysis indicated a considerable proportion of the women need to have permission from their fathers or husbands to practice the PA outside of the home (in a public area or in a gym) $(74.2 \%)$ or to have and buy any exercise equipment (62.9\%). Since the Saudi women are prohibited from driving, a large proportion of the women reported that their family did not allow them to use public transportation services if they went out home to practice the PA (72.7\%), or they were not allowed to have friends pick them to take them to or from the gym (41.4\%). Furthermore, social environmental barriers to PA were significantly higher among adolescents ages $15-19$. This relationship could be due to $74.9 \%$ of the adolescents unmarried and living with their parents or guardians. These findings highlight the great impact of the conservative Saudi society, norms, and politics on women's PA levels. Therefore, to help those women be more active and have a healthy life, efforts should be concentrated to mitigate these social barriers and norms.

Additionally, the data revealed a high proportion of the women had faced physical environmental barriers to PA (56.6\%). The sum score of physical environmental barriers subgroups to PA (lack of resources, lack of transportation, and hot weather barriers) showed that the most important barriers for women across those barriers subgroups was the hot weather in Jeddah, which prevented them for exercising outdoors. Also, Musaiger (2014) found that $58.7 \%$ of the young Kuwaiti women (age 19-26) had barriers to practicing PA due to the hot weather [52]. It is well known that Saudi Arabia and the Arab Gulf countries all have long (six months) and hot summer season $\left(43^{\circ} \mathrm{C}, 109^{\circ} \mathrm{F}\right)$. Therefore, the chance for outdoor PA or sports during the year is limited for Saudi and Arab Gulf people. Furthermore, in the present study we found a considerable proportion of the women reported 
that the absence of walking track in the malls (indoor mall walking track roof with air conditioner) prevented them from walking during the summertime (86.9\%). When considering the lack of PA resources and transportation, $60.2 \%$ of the women reported that walking in their neighborhood was unsafe, 56.5\% reported there was a lack of access to PA facilities (e.g., jogging trails, sidewalks, or swimming pools), and 54\% had difficulty finding transportation to go outside the home to exercise. These findings were not surprising, since in Saudi Arab, women are prohibited from driving and practicing PA at the public schools, and there is limited access to join gym clubs or walking outdoors in the hot temperature [15]. As with the social environmental barrier to PA, the physical environmental barrier to PA was significantly higher among adolescents, age 15-19. This association also could be due to those women being unmarried and living with their parents or guardians and needing their permission to access a PA facility to practice the PA. In order to facilitate the promotion of PA in Saudi Arabia, specific gender-based barriers should be taken into consideration when making recommendations to promote PA, which means that intervention and motivation programs should be customized to suit the needs of the individual, with gender as a primary consideration.

\section{Relationship between perceived barriers to maintaining healthy body weight and eating habits (EHs) and physical activity (PA)}

A systematic review of peer-reviewed studies of PA among adults found a positive association between EHs and PA [63]. AlHazzaa (2014) found a positive significant association for many healthful dietary habits with PA among 2822 Saudi adolescents (15-19 years old, 51\% female) conducted in three major cities in Saudi Arabia (Al-khober, Al-Riyadh, and Jeddah) [64]. The results of the current study, in agreement with those findings, found a positive significant association between EHs and PA among the study population.

Multiple personal, social, and physical environmental barriers influence the EHs and PA in women $[5,39,52,56]$. The current study findings confirmed the relationships between EHs and these barriers. There was a significant inverse association between the women's EHs and overall HE barriers and the HE categories barriers: personal, and social environmental barriers. However, there were no significant associations found between PA level, overall PA barriers, and the PA subgroup barriers: personal, social, and physical environmental barriers. While a review of the correlations of PA in adults demonstrated a significant inverse association between the PA and the environmental and personal barriers, the magnitude of their association with physical inactivity depends on the population studied [63]. Moreover, in a convenience sample of 285 Hispanic/Latino women age 20-50 living in Northern Virginia, USA, the social environmental barrier had a significant negative association with PA, while personal and physical environmental barriers were not significantly associated with PA [65]. One possible explanation for the divergent findings may be due to the high proportion of women in the current present study who suffered from PA barriers, even if they were active. The data revealed $52.1 \%(n=145)$ of the active women faced PA barriers.

\section{Association between obesity and barriers to maintaining healthy body weight}

This study found a relatively high prevalence of overweight and obesity among the study population, and only $26.6 \%$ (108) of the women fell within normal or healthy weight. However, no significant differences were found between obese and non-obese women (BMI and WC) regarding the overall barriers score to maintaining healthy body weight (HE or PA barriers). These findings are in agreement with a previous study's finding that investigated the barriers to weight maintenance among 530 university students (203 men and 327 women), ages 19-26, in Kuwait [52]. They did not find any significant associations between obese and nonobese women regarding barriers to HE and PA. This finding could be due to the highly prevalent perceived barriers to maintaining healthy body weight among our study population even among women with normal weight.

\section{Study limitation}

The study had a few limitations. First, the cross-sectional nature of the study would not allow for cause-effect relationships to be established. Second, the questionnaire assessments of PA are subject to recall bias, and the self-reported PA did not provide accurate estimates of absolute amounts of activity (Metabolic Equivalent Task minutes per week). A third limitation was that the results of the study could only be generalized to non-pregnant Saudi women of reproductive age (15-49 years) who obtained services at PHCCs in Jeddah City, and were not applicable to all Saudi women living in Jeddah or other cities in Saudi Arabia. Therefore, replication of this study in different populations or in different cities in Saudi Arabia (such as rural or mountainous areas) is highly suggested to allow for comparisons between study results. Comparison between studies results may provide different data and different recommendations that help to develop appropriate and effective obesity prevention strategies for Saudi women.

\section{Conclusion}

The current study's data show that obesity, unhealthy eating habits, and physical inactivity are major health problems in Saudi women in Jeddah. Findings from this study demonstrated that there were several personal, personal, social, and physical environmental barriers related to HE and PA facing Saudi women in Jeddah. To our knowledge, this is also the first study to explore a range of personal, social, and physical environmental factors that act as barriers to maintaining a healthy weight through healthy EHs and PA levels among the study population. Determining these factors and barriers is vital to creating effective programs for combating obesity not only among the women of Jeddah City but also, potentially, women in other Saudi Arabian cities. Barriers related to the physical environment had the greatest effect on $\mathrm{HE}$ and PA, with significant associations between overall HE barriers score and 
age, and between overall PA barriers score and income level. Adolescents faced the most barriers to eating healthy foods, while women with low incomes faced the most barriers to engaging in PA. As well, low-income and married women were the most likely to experience personal barriers to eating healthy foods, while women with low incomes and low education levels were the most likely to report personal barriers to engaging in PA. Adolescents were also highly influenced by social and physical environmental barriers to be inactive. These findings suggest that community-based interventions and public health strategies should concentrate on adolescents and women with low incomes. The most common barriers to HE and PA in the study group were lack of willpower, skills, knowledge, enjoyment, time, and resources, as well as social influences. Efforts should therefore focus on mitigating these personal, physical environmental, and social barriers. In addition, we found that hot weather and the absence of walking tracks in malls stopped women from walking or exercising during summer. Thus, we recommend creating air-conditioned walking tracks inside malls to encourage PA during summer. Ultimately, such information can be used to design gender- and culture-sensitive interventions that could enhance adherence to EHs and PA that are relevant to the Saudi society.

\section{Acknowledgments}

The authors wish to thank the Jeddah Primary Health Centers (JPHCCs) that provided us the opportunity to conduct research at their facilities. We gratefully acknowledge the assistance of Professor James M. Lepkowski of the University of Michigan's Department of Biostatistics in helping design and select the sample of clinics, design the sample of eligible women within clinics, and develop suitable complex sample design estimation procedures, including nonresponse adjusted sampling weights.

\section{References}

1. Flegal KM, Carroll MD, Ogden CL, Curtin LR (2010) Prevalence and trends in obesity among US adults, 1999-2008. JAMA 303: 235-41.

2. Memish ZA, El Bcheraoui C, Tuffaha M, Robinson M, Daoud F, et al. (2013) Obesity and associated factors Kingdom of Saudi Arabia, 2013. Prev Chronic Dis 11: E174.

3. Daoud F, El Bcheraoui C, Tuffaha M, AlMazroa MA, Al Saeedi M, et al. (2016) The health status of Saudi women: findings from a national survey. J Public Health (Oxf) 38: 660-72.

4. Çitozi R, Bozo D, Pano G (2013) An assessment of the perception of physical activity, eating habits, self-efficacy and the knowledge about healthy food in Albanian adolescents. J of Human Sport and Exerc 8: 192-203.

5. Andajani-Sutjahjo S, Ball K, Warren N, Inglis V, Crawford D (2004) Perceived personal, social and environmental barriers to weight maintenance among young women: A community survey. Int J Behav Nutr Phys Act 1: 15.

6. Timmerman GM (2007) Addressing barriers to health promotion in underserved women. Fam Community Health 30: S34-42.

7. Ali HI, Baynouna LM, Bernsen RM (2010) Barriers and facilitators of weight management: perspectives of Arab women at risk for type 2 diabetes. Health Soc Care Community 18: 219-28.

8. Benazeera UJC (2014) Association between EHs and body mass index (BMI) of adolescents. Int J Med Sci Public Health 3: 940-43.

9. Michaelidou N, Christodoulides G, Torova K (2012) Determinants of healthy eating: a cross-national study on motives and barriers. Int J Consum Stud 36: $17-22$.

10. Al-Jaaly E, Lawson M, Hesketh T (2011) Overweight and its Determinants in Adolescent Girls in Jeddah City, Saudi Arabia. Int J Food Nut Publ Health 4: 95-108.

11. Jakicic JM, Otto AD (2005) Physical activity considerations for the treatment and prevention of obesity. Am J Clin Nutr 82: 226S-9S.

12. Anokye NK, Trueman P, Green C, Pavey TG, Taylor RS (2012) Physical activity and health related quality of life. BMC Public Health 12: 624.

13. McGuire S (2011) U.S. Department of Agriculture and U.S. Department of Health and Human Services, Dietary Guidelines for Americans, 2010. 7th Edition, Washington, DC: U.S. Government Printing Office, January 2011. Adv Nutr 2: 293-4.

14. Al-Hazzaa HM (2007) Health-enhancing physical activity among Saudi adults using the International Physical Activity Questionnaire (IPAQ). Public Health Nutr 10: 59-64.

15. Al-Eisa ES, Al-Sobayel HI (2012) Physical Activity and Health Beliefs among Saudi Women. J Nutr Metab 2012: 642187.

16. Hillemeier MM, Weisman CS, Chuang C, Downs DS, McCall-Hosenfeld J, et al. (2011) Transition to overweight or obesity among women of reproductive age. J Womens Health (Larchmt) 20: 703-10.

17. Khashoggi RH, Madani KA, Ghaznawy HI, Ali MA (1994) Socioeconomic factors affecting the prevalence of obesity among female patients attending primary health centers in Jeddah, Saudi Arabia. Ecol Food Nutr 31: 277-83.

18. World Health Organization (2012) Global Database on Body Mass Index. BMI classification, Switzerland.

19. de Onis M, Onyango AW, Borghi E, Siyam A, Nishida C, et al. (2007) Development of a WHO growth reference for school-aged children and adolescents. Bull World Health Organ 85: 660-7.

20. World Health Organization (2010) Anthro plus for personal computers, version 3.3.2, 2011: Software for assessing growth and development of the world's children. Geneva, Switzerland.

21. World Health Organization (2011) Waist circumference and waist-hip ratio: Report of a WHO expert consultation, Geneva, 8-11, Switzerland.

22. Turconi G, Guarcello M, Maccarini L, Cignoli F, Setti S, et al. (2008) Eating habits and behaviors, physical activity, nutritional and food safety knowledge and beliefs in an adolescent Italian population. J Am Coll Nutr 27: 31-43.

23. Turconi G, Celsa M, Rezzani C, Biino G, Sartirana MA, et al. (2003) Reliability of a dietary questionnaire on food habits, eating behaviour and nutritional knowledge of adolescents. Eur J Clin Nutr 57: 753-63.

24. Al-Rethaiaa AS, Fahmy AE, Al-Shwaiyat NM (2010) Obesity and eating habits among college students in Saudi Arabia: a cross sectional study. Nutr J 9: 39.

25. Soori H (2001) Pattern of dietary behaviour and obesity in Ahwaz, Islamic Republic of Iran. East Mediterr Health J 7: 163-70.

26. Rasheed P (1998) Perception of body weight and self-reported eating and exercise behaviour among obese and non-obese women in Saudi Arabia. Public Health 112: 409-14.

Annex Publishers | www.annexpublishers.com

Volume 3 | Issue 2 
27. Al-Othaimeen AI, Al-Nozha M, Osman AK (2007) Obesity: an emerging problem in Saudi Arabia. Analysis of data from the National Nutrition Survey. East Mediterr Health J 13: 441-8.

28. Al Qauhiz NM (2010) Obesity among Saudi Female University Students: Dietary Habits and Health Behaviors. J Egypt Public Health Assoc 85: 45-59.

29. Musaiger AO (2004) Overweight and obesity in the Eastern Mediterranean Region: can we control it? East Mediterr Health J 10: 789-93.

30. Dewar DL, Lubans DR, Plotnikoff RC, Morgan PJ (2012) Development and evaluation of social cognitive measures related to adolescent dietary behaviors. Int J Behav Nutr Phys Act 9: 36.

31. International Physical Activity Questionnaire (2002) Stockholm, Karolinska Institutet, Denmark.

32. Craig CL, Marshall AL, Sjöström M, Bauman AE, Booth ML, et al. (2003) International physical activity questionnaire: 12-country reliability and validity. Med Sci Sports Exerc 35: 1381-95.

33. AboZaid HA, Farahat FM (2010) Physical activity profile among patients attending family medicine clinics in western Saudi Arabia. Saudi Med J $31: 428-33$.

34. Al-Hazzaa HM (2004) The public health burden of physical inactivity in Saudi Arabia. J Family Community Med 11: 45-51.

35. Abdelbaky A, Abozaid H, Alsahafi AA, Hendi OM, Amin OA, et al. (2017) Physical activity among Taif university students at Taif governorate, Saudi Arabia. World J Pharmaceutical Med Res 3: 12-7.

36. International Physical Activity Questionnaire (2005) Guidelines for data processing and analysis of the International Physical Activity Questionnaire (IPAQ).

37. Centers for Disease Control and Prevention (1999) Barriers to Being Physically Active Quiz, US Department of Health and Human Services, USA.

38. Samir N, Mahmud S, Khuwaja AK (2011) Prevalence of physical inactivity and barriers to physical activity among obese attendants at a community health-care center in Karachi, Pakistan. BMC Res Notes 4: 174.

39. AlQuaiz AM, Tayel SA (2009) Barriers to a healthy lifestyle among patients attending primary care clinics at a university hospital in Riyadh. Ann Saudi Med 29: 30-5.

40. Lappalainen R, Saba A, Holm L, Mykkanen H, Gibney MJ, et al. (1997) Difficulties in trying to eat healthier: descriptive analysis of perceived barriers for healthy eating. Eur J Clin Nutr 51: S36-40.

41. Brownson RC, Baker EA, Housemann RA, Brennan LK, Bacak SJ (2001) Environmental and policy determinants of physical activity in the United States. Am J Public Health 91: 1995-2003.

42. Ziebland S, Thorogood M, Yudkin P, Jones L, Coulter A (1998) Lack of willpower or lack of wherewithal? "Internal" and "external" barriers to changing diet and exercise in a three year follow-up of participants in a health check. Soc Sci Med 46: 461-5.

43. Daskapan A, Tuzun EH, Eker L (2006) Perceived Barriers to Physical Activity in University Students. J Sports Sci Med 5: 615-20.

44. Dutton GR, Johnson J, Whitehead D, Bodenlos JS, Brantley PJ (2005) Barriers to physical activity among predominantly low-income African-American patients with type 2 diabetes. Diabetes Care 28: 1209-10.

45. Gilbert DT, Fiske ST, Lindzey G (1998) Social influence: Social norms, conformity, and compliance In: handbook of social psychology (4 $4^{\text {th }}$ Editon) New York: McGraw-Hill, USA.

46. Centers for Disease Control and Prevention (1996) U.S. Department of Health and Human Services, Physical activity and health: a report of the surgeon general. Atlanta, USA.

47. Barnhart JM, Wright ND, Freeman K, Silagy F, Correa N, et al. (2009) Risk perception and its association with cardiac risk and health behaviors among urban minority adults: the Bronx Coronary Risk Perception study. Am J Health Promot 23: 339-42.

48. Lai WT, Chen CF (2011) Behavioral intentions of public transit passengers-The roles of service quality, perceived value, satisfaction and involvement. Transport Policy 18: 318-25.

49. Andreou E, Alexopoulos EC, Lionis C, Varvogli L, Gnardellis C, et al. (2011) Perceived Stress Scale: reliability and validity study in Greece. Int J Environ Res Public Health 8: 3287-98.

50. British Nutrition Foundation (2015) Healthy hydration guide, London.

51. Samara A, Nistrup A, Al-Rammah TY, Aro AR (2015) Lack of facilities rather than sociocultural factors as the primary barrier to physical activity among female Saudi university students. Int J Womens Health 7: 279-86.

52. Musaiger AO, Al-Kandari FI, Al-Mannai M, Al-Faraj AM, Bouriki FA, et al. (2014) Perceived barriers to weight maintenance among university students in Kuwait: the role of gender and obesity. Environ Health Prev Med 19: 207-14.

53. Musaiger AO, Al-Mannai M, Tayyem R, Al-Lalla O, Ali EY, et al. (2013) Perceived barriers to healthy eating and physical activity among adolescents in seven Arab countries: a cross-cultural study. ScientificWorldJournal 2013: 232164.

54. Al-Otaibi HH (2013) Measuring stages of change, perceived barriers and self efficacy for physical activity in Saudi Arabia. Asian Pac J Cancer Prev 14: 1009-16. 55. Crawford D, Ball K (2002) Behavioural determinants of the obesity epidemic. Asia Pacific J Clin Nutr 11: S718-21.

56. Al-Farwan WM (2011) Perceived Personal, Social, and Environmental Barriers to Healthy Eating Among Young Overweight and Obese Saudi Women. Middle East J Fam Med 9: 3-9.

57. Kelly S, Martin S, Kuhn I, Cowan A, Brayne C, et al. (2016) Barriers and Facilitators to the Uptake and Maintenance of Healthy Behaviours by People at MidLife: A Rapid Systematic Review. PLoS One 11: e0145074.

58. Escoto KH, Laska MN, Larson N, Neumark-Sztainer D, Hannan PJ (2012) Work Hours and Perceived Time Barriers to Healthful Eating Among Young Adults. Am J Health Behav 36: 786-96.

59. Wang ML, Pbert L, Lemon SC (2014) Influence of family, friend and coworker social support and social undermining on weight gain prevention among adults. Obesity (Silver Spring) 22: 1973-80.

60. Sequeira S, Cruz C, Pinto D, Santos L, Marques A (2012) Prevalence of barriers for physical activity in adults according to gender and socioeconomic status. Br J Sports Med 45: A18-19.

61. Scheerder J, Thomis M, Vanreusel B, Lefevre J, Renson R, et al. (2006) Sports Participation Among Females From Adolescence To Adulthood. Int Rev Sociol Sport 41: 413-30.

62. Sebastião E, Chodzko-Zajko W, Schwingel A, Gobbi LTB, Papini CB, et al. (2013) Perceived barriers to leisure time physical activity: What Brazilians have to say? Open J Prev Med 3: 491-9. 
63. Reichert FF, Barros AJ, Domingues MR, Hallal PC (2007) The role of perceived personal barriers to engagement in leisure-time physical activity. Am J Public Health 97: 515-9.

64. Al-Hazzaa HM, Al-Sobayel HI, Abahussain NA, Qahwaji DM, Alahmadi MA, et al. (2014) Association of dietary habits with levels of physical activity and screen time among adolescents living in Saudi Arabia. J Hum Nutr Diet 27: 204-13.

65. Voorhees CC, Rohm Young D (2003) Personal, social, and physical environmental correlates of physical activity levels in urban Latinas. Am J Prev Med 25: 61-8.

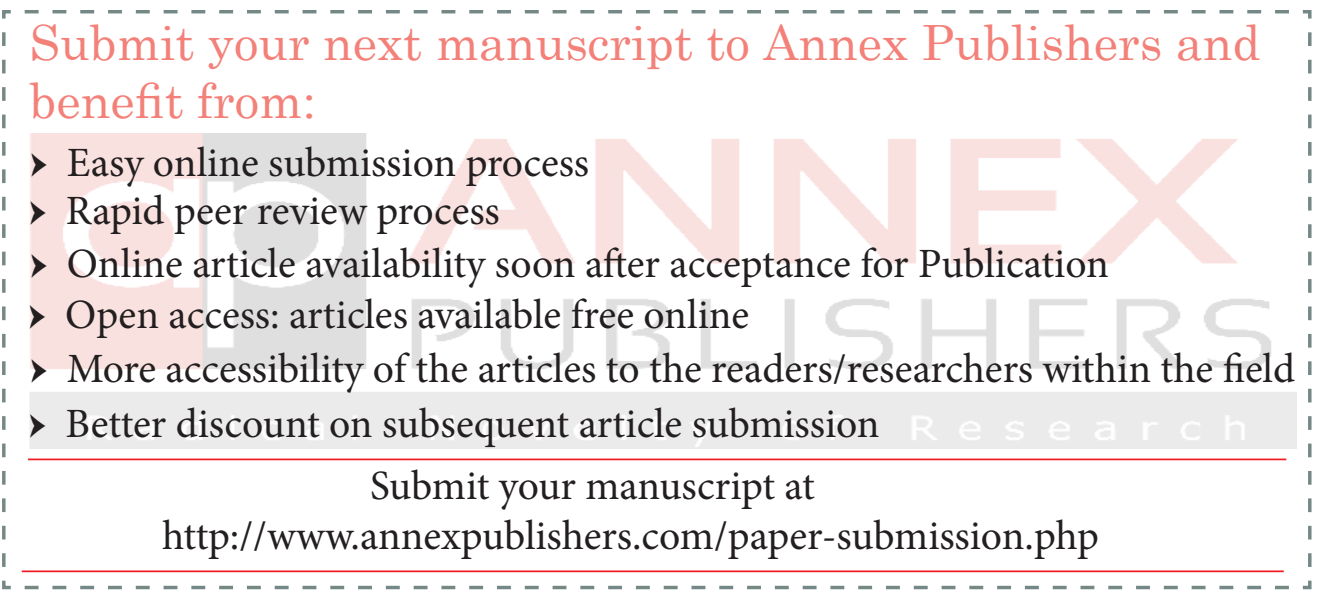

